\title{
Efficient estimation of analytic density under random censorship
}

\author{
EDUARD BELITSER
}

Department of Mathematics, Free University Amsterdam, de Boelelaan 1081a, $1081 \mathrm{HV}$ Amsterdam, the Netherlands.E-mail: belitser@cs.vu.nl

The nonparametric minimax estimation of an analytic density at a given point, under random censorship, is considered. Although the problem of estimating density is known to be irregular in a certain sense, we make some connections relating this problem to the problem of estimating smooth functionals. Under condition that the censoring is not too severe, we establish the exact limiting behaviour of the local minimax risk and propose the efficient (locally asymptotically minimax) estimator-an integral of some kernel with respect to the Kaplan-Meier estimator.

Keywords: asymptotic local minimax risk; density estimation; Kaplan-Meier estimator; kernel; random censorship

\section{Introduction}

Let $\left(X_{1}, Y_{1}\right), \ldots,\left(X_{n}, Y_{n}\right)$ be independent identically distributed pairs of random variables where $X_{1}$ and $Y_{1}$ are independent and have the distribution functions $F$ and $G$, respectively. We assume also that the distribution of $X_{1}$ is absolutely continuous with density $f$. The following model is known as random censorship model. We observe only the pairs $\left(Z_{i}, \Delta_{i}\right)$, $i=1,2, \ldots, n$, with $Z_{i}=\min \left(X_{i}, Y_{i}\right)$ and $\Delta_{i}=I\left\{X_{i} \leqslant Y_{i}\right\}$. In survival analysis the $X_{i}$ are called survival times and the $Y_{i}$ censoring times. Estimation problems with censored observations arise often in lifetime research. In survival analysis, lifetime data are typically subject to censoring. We suppose $F$ and $G$ are unknown and our goal is, using the observed data, to estimate the density $f(x)$ at a given point $x$.

The problem of density estimation under random censorship has been treated by a number of authors (see, for example, Mielniczuk (1986), Diehl and Stute (1988), Lo et al. (1989), Hentzschel (1992), Huang and Wellner (1995), Kulasekera (1995) and Liu (1996)). Hentzschel (1992) investigated the estimator based on the orthonormal system of the Laguerre series on the positive line and under some assumptions obtained the rates of the mean integrated square error and the mean square error. Kulasekera (1995) gave the upper $L_{1}$ bounds for the kernel-type estimator for two classes of densities: monotonically decreasing densities on $[0, \infty)$ and densities which are of bounded variation on $[0,1]$. For a decreasing density function, Huang and Wellner (1995) showed that the nonparametric maximum-likelihood estimator of the density is asymptotically equivalent to the estimator 
obtained by differentiating the least-concave majorant of the Kaplan-Meier estimator and established the asymptotic distributions of the different estimators at a fixed point.

However, in all the above-mentioned papers the question of optimality has not been touched on. In a recent paper, Liu (1996) considered the general problem of estimating functionals of a distribution $F$ for some nonparametric classes defined in terms of the Hellinger modulus of continuity. With regard to density estimation, minimax Kaplan-Meierbased kernel procedures were shown, under some conditions, to be of optimal rate and, moreover, within certain lower and upper bounds.

In the nonparametric minimax estimation context the notion of asymptotic optimality is usually associated with the optimal rate of convergence of the minimax risk. In order to derive the exact asymptotics of the minimax risk and to be able to compare the estimators with the optimal rate of convergence, one may strengthen the optimal rate results by finding optimal constants when they exist. Results about the optimal constants in minimax density estimation have only been obtained in a limited number of studies for models with independent identically distributed observations. The majority of workers have considered the global minimax risk. However, studying the so-called local minimax risk yields more exact results. We mention the work of Golubev and Levit (1996) whose results motivated the present study. In the problem of estimation of an analytic density at a given point, with independent identically distributed observations, they derived the exact limiting behaviour of the local minimax risk and proposed an efficient estimator.

To elucidate the ideas of the results, we give here some heuristic arguments. The unknown underlying density $f$ is assumed to belong to the class of densities with exponentially decreasing Fourier transforms (analytic densities). This nonparametric class has the advantage that one can treat the problem of estimating $f(x)$ as if a smooth functional was to be estimated. In particular, it turns out that any density from this nonparametric class can be represented in the following asymptotic form (see Lemma 5 below):

$$
f(x)=\int \phi_{n}(x-y) f(y) \mathrm{d} y+O\left(n^{-1 / 2}\right), \quad \text { as } n \rightarrow \infty,
$$

locally uniformly in $f$ in a proper topology, where $\phi_{n}(y)$ is some sequence of functions (see the exact definitions in the next section) which we shall call kernel, treating this notion in a broader sense than is usual in the literature. The local minimax quadratic risk proves to be of order $(\log n) / n$ and therefore the remainder term can be neglected, while the first term resembles a "smooth" functional (it is a sequence of functionals because of its dependence on $n$ ) to which one can apply well-developed methods for deriving an optimal estimator and its asymptotic behaviour. So, in case there is no censoring, one can expect the estimator $\tilde{f}_{n}(x)=\int \phi_{n}(x-y) \mathrm{d} F_{n}(y)$, with the empirical distribution function $F_{n}$, to be optimal in some sense. If for independent identically distributed observations the estimator of the density is some functional of the empirical distribution function $T\left(F_{n}\right)$, then in the case of censoring one tries usually to use the estimator $T\left(\tilde{F}_{n}\right)$, with the well-known Kaplan-Meier estimator $\tilde{F}_{n}(y)$ (see below) instead of the empirical distribution function $F_{n}$. Thus, it is natural to propose the estimator

$$
\tilde{f}_{n}=\tilde{f}_{n}(x)=\int \phi_{n}(x-y) \mathrm{d} \tilde{F}_{n}(y)
$$


In this paper we establish, under the condition that the censoring is not too severe, the exact limiting behaviour of the local minimax risk up to a constant and show that the estimator of the form (1), with a properly chosen kernel, is locally asymptotically efficient. We emphasize here that the choice of nonparametric class (analytic densities) has made it possible. We propose a wide class of kernels on which the estimator can be based, which turns out to be important in the estimation problem with censored observations. Using the martingale approach enables us to derive the exact upper bound for the local minimax risk. The lower bound for the local minimax risk is based on the elementary van Trees inequality (Gill and Levit 1995).

\section{Definitions and main results}

In this section we summarize the main results. First we define the notion of efficiency.

Prior information about an unknown density $f$ is usually formalized by assuming $f \in \mathscr{F}$, for some class of densities $\mathscr{F}$. Suppose now that we have some topology on $\mathscr{F}$. For each neighbourhood $V$ define the local minimax risk:

$$
r_{n}(V)=r_{n}(V, x)=\inf _{\tilde{f}_{n}} \sup _{f \in V} \mathrm{E}_{f}\left\{\tilde{f}_{n}(x)-f(x)\right\}^{2},
$$

where the infimum is taken over all estimators $\tilde{f}_{n}$. The most convenient and natural way to introduce the notion of efficiency is the following: the estimator $\tilde{f}_{n}$ is called asymptotically efficient (or just efficient) if for each density $f \in \mathscr{F}$ there exists a neighbourhood $V_{0} \ni f$ such that, for any neighbourhood $V, f \in V \subseteq V_{0}$ (from now on we shall just say for any sufficiently small neighbourhood of $f$ ), for some positive sequence $\beta_{n}$,

$$
\limsup _{n \rightarrow \infty} \beta_{n}^{-2}\left(\sup _{f \in V} \mathrm{E}_{f}\left\{\tilde{f}_{n}(x)-f(x)\right\}^{2}-r_{n}(V)\right)=0,
$$

while

$$
\liminf _{n \rightarrow \infty} \beta_{n}^{-2} r_{n}(V)>0
$$

The sequence $\beta_{n}$ is called the minimax rate of convergence. Note also that one can write $\lim$ instead of lim sup. If the limit

$$
\lim _{V \downarrow f_{0}} \lim _{n \rightarrow \infty} \beta_{n}^{-2} r_{n}(V)=\sigma^{2}\left(f_{0}\right)
$$

exists, then $\sigma\left(f_{0}\right)$, together with $\beta_{n}$, describes the exact behaviour of the local minimax risk and represents in a way the difficulty of the estimation problem at the point $f_{0}$.

Denote from now on the Fourier transform of an absolutely integrable function $f$ by $\hat{f}$ :

$$
\hat{f}(t)=\int \mathrm{e}^{\mathrm{i} t y} f(y) \mathrm{d} y .
$$

Define now the nonparametric class $\mathscr{F}_{\delta}$ of underlying densities. 
Definition. For given $P, \delta>0$, denote

$$
\mathscr{F}_{\delta}=\mathscr{F}_{\delta}(P)=\left\{f(\cdot):(2 \pi)^{-1} \int \cosh ^{2}(\delta t)|\hat{f}(t)|^{2} \mathrm{~d} t \leqslant P\right\} .
$$

Remark 1. This is a class of analytic functions. Below we describe it more precisely. Let the class $\mathscr{b}_{\delta}=\mathscr{b}_{\delta}(P)$ consist of functions admitting bounded analytic continuation into the strip $\{y+\mathrm{i} u,|u| \leqslant \delta\}$ and $\int|f(y+\mathrm{i} \delta)|^{2} \mathrm{~d} y \leqslant P<\infty$. In the case where there is no censoring the nonparametric classes of the type $\mathscr{b}_{\delta}$ were considered first by Ibragimov and Hasminski (1983), where the minimax rates of convergence in $L_{p}$ were derived. There is a close relationship between the classes $\mathscr{F}_{\delta}$ and $\mathscr{A}_{\delta}$; for any $\beta, 0<\beta<\delta$, there exists $Q>0$ such that

$$
\mathscr{C}_{\delta}(P) \subseteq \mathscr{F}_{\delta}(P) \subseteq \mathscr{C}_{\delta-\beta}(Q)
$$

Indeed, if a density $f \in A_{\delta}$, then, according to Timan (1963, p. 137), the limit

$$
\lim _{u \rightarrow \delta} \operatorname{Re}\{f(y+\mathrm{i} u)\}=g_{f}(y)
$$

exists for almost all $y$ and $f(y)$ can be represented as a convolution:

$$
f(y)=\frac{1}{2 \delta} \int\left\{\cosh \left(\frac{\pi(y-u)}{2 \delta}\right)\right\}^{-1} g_{f}(u) \mathrm{d} u .
$$

Furthermore, because of the relation (see, for example, Gradshtein and Ryzhik (1980, equation (3.983.1)))

$$
\begin{gathered}
\frac{1}{2 \delta} \int \mathrm{e}^{\mathrm{i} t u}\left\{\cosh \left(\frac{\pi u}{2 \delta}\right)\right\}^{-1} \mathrm{~d} u=\{\cosh (\delta t)\}^{-1}, \\
\hat{f}(t)=\{\cosh (\delta t)\}^{-1} \hat{g}(t) .
\end{gathered}
$$

By the Parseval formula,

$$
\frac{1}{2 \pi} \int \cosh ^{2}(\delta t)|\hat{f}(t)|^{2} \mathrm{~d} t=\int g_{f}^{2}(y) \mathrm{d} y \leqslant P,
$$

and hence the first inclusion holds. The second inclusion follows immediately from the Paley-Wiener theorem (see, for example, Katznelson (1976, p. 174)).

Note also that the class $\mathscr{F}_{\delta}$ is quite broad; the Gauss, Student and Cauchy distributions are, among many others, for appropriate $\delta$, in this class, as well as their mixtures.

Definition. Let $\mathscr{T}_{\delta}$ be the topology on $\mathscr{F}_{\delta}$ induced by the distance

$$
\rho(f, g)=\left(\int \cosh ^{2}(\delta t)|\hat{f}(t)-\hat{g}(t)|^{2} \mathrm{~d} t\right)^{1 / 2}+\int|f(y)-g(y)| \mathrm{d} y .
$$

Remark 2. This is a strong topology; closeness with respect to $\rho$ implies, by the formula for 
the inverse Fourier transform, closeness of all derivatives in the uniform topology: for $g$, $h \in \mathscr{F}_{\delta}$,

$$
\sup _{y}\left|g^{(m)}(y)-h^{(m)}(y)\right| \leqslant C_{1}\left(\int t^{2 m}|\hat{g}(t)-\hat{h}(t)|^{2} \mathrm{~d} t\right)^{1 / 2} \leqslant C_{2} \rho(g, h) .
$$

In almost every estimation problem with censored data, one faces the well-known unstable behaviour of the Kaplan-Meier process $n^{1 / 2}\left\{\tilde{F}_{n}(y)-F(y)\right\}$ (here $\tilde{F}_{n}$ is the Kaplan-Meier estimator; see definition below) in the right tails of $F$ and $G$. Therefore, the lighter the tails of the kernel, the less restrictive conditions on the censoring mechanism are needed. On the other hand, it turns out that, when constructing an efficient estimator, one has to use observations distant from $x$ as well as those close to $x$. Roughly speaking, this corresponds to the fact that, even for $y$ values distant from $x$, the values $f(y)$ still carry some information about $f(x)$; analytic functions have a "long memory". This is formalized by imposing the following restriction on the nonparametric class $\mathscr{F}_{\delta}$.

Definition. For given $\alpha, \tau_{0}>0, m \geqslant 1$, denote

$$
\tilde{\mathscr{F}}_{\delta}=\tilde{\mathscr{F}}_{\delta}(m)=\mathscr{F}_{\delta}(P) \cap\left\{f: \inf _{y}\left[\mathrm{e}^{\tau_{0} y^{2 m}}\{1-F(y)\}\{1-G(y)\}\right]>\alpha\right\},
$$

where $\mathscr{F}_{\delta}(P)$ is defined by (3) and $F$ is the distribution function corresponding to the density $f$.

Remark 3. The restriction on the original class $\mathscr{F}_{\delta}$ defined by (3) expresses the requirement for the censoring mechanism to allow distant observations with positive probability as the number of observations tends to infinity. Indeed, for some $0<p<1$ let

$$
y_{n}=\left\{\frac{1}{\tau_{0}} \log \left(\frac{-\alpha n}{\log (1-p)}\right)\right\}^{1 / 2 m}
$$

then

$$
\begin{aligned}
\mathbf{P}\left\{Z_{(n)}>y_{n}\right\} & =1-\left[1-\left\{1-F\left(y_{n}\right)\right\}\left\{1-G\left(y_{n}\right)\right\}\right]^{n} \\
& \geqslant 1-\left(1-\alpha \mathrm{e}^{-\tau_{0} y_{n}^{2 m}}\right)^{n} \\
& =1-\left(1+\frac{\log (1-p)}{n}\right)^{n} \\
& \geqslant p,
\end{aligned}
$$

where $Z_{(n)}=\max _{1 \leqslant i \leqslant n} Z_{i}$.

Remark 4. Without loss of generality we suppose that $m$ is integer. Indeed, we shall see later that both the upper bound and the lower bound for the local minimax risk do not depend on $m$, nor on $\alpha, P$ and $\tau_{0}$. 
Definition. Let $\tilde{\mathscr{T}}_{\delta}$ be the topology induced by $\mathscr{T}_{\delta}$ on $\tilde{\mathscr{F}}_{\delta}$.

Let us establish several conventions; sometimes we shall write $F \in \tilde{\mathscr{F}}_{\delta}$, meaning actually that the corresponding density $f \in \tilde{\mathscr{F}}_{\delta}$; in the proofs we shall denote generic positive constants by $C_{1}, C_{2}, C_{3}, \ldots$ and they are assumed to be different in the proofs of different assertions; all symbols $O$ and $o$ correspond to the asymptotics $n \rightarrow \infty$ unless otherwise specified; if we say that a particular property holds locally uniformly, this means that for each $f \in \tilde{\mathscr{F}}_{\delta}$ there exists a neighbourhood $V$ of $f$ such that this property holds uniformly over $V$.

We propose the following class of kernels to be used in the construction of the estimator:

$$
\phi_{n}(y)=\phi_{n}(y, \tau, \delta, m)=r(y) s(y),
$$

where

$$
r(y)=r(\tau, m, y)=\mathrm{e}^{-\tau y^{2 m}}, \quad s(y)=s(\delta, n, y)=\frac{\sin \left(a_{n} y\right)}{\pi y}, \quad a_{n}=\frac{\log n}{2 \delta}
$$

and $\tau$ is any fixed number such that $\tau>\frac{3}{2} \tau_{0}$, where constant $\tau_{0}$ appears in the definition of the class $\tilde{\mathscr{F}}_{\delta}$.

Let us state some properties of kernels of the form (5) and (6):

$$
\begin{gathered}
\hat{\phi}_{n}(t)=\frac{1}{2 \pi}\left(\hat{r} * I_{\left(-a_{n}, a_{n}\right)}\right)(t), \\
\int \phi_{n}^{2}(y) \mathrm{d} y=\frac{a_{n}}{\pi}\{1+o(1)\}=\frac{\log n}{2 \pi \delta}\{1+o(1)\},
\end{gathered}
$$

where $*$ is the convolution operation and $I_{S}$ denotes the indicator function of set $S$. The first property is merely application of the standard formula for the Fourier transform of the product of two functions. To get the second relation, split the integral into two terms: integral over small neighbourhood of 0 and integral over its complement. Further note that the first term is asymptotically equivalent to

$$
a_{n} \pi^{-2} \int\left(\frac{\sin y}{y}\right)^{2} \mathrm{~d} y=\frac{a_{n}}{\pi}
$$

and the second is of order $O(1)$.

Note also that the function $\hat{r}(t)$ is even. The asymptotic behaviour of $\hat{r}(t)$, as $|t| \rightarrow \infty$, has been described by Fedoruk (1977, pp. 213-214, 220). We adapt this result in simplified form, suitable for our purposes; for some $A_{1}, A_{2}>0$,

$$
|\hat{r}(t)| \leqslant A_{1} \exp \left(-A_{2}|t|^{2 m /(2 m-1)}\right) .
$$

The constants $A_{1}, A_{2}$ depend in general on $m$ and $\tau$.

Define now the following estimator:

$$
\tilde{f}_{n}=\tilde{f}_{n}(x)=\int \phi_{n}(x-y) \mathrm{d} \tilde{F}_{n}(y)
$$

where $\phi_{n}(y)$ is defined by (5) and (6) and $\tilde{F}_{n}(y)$ is the Kaplan-Meier estimator, a well- 
known nonparametric efficient estimator of the distribution function $F(y)$ (see, for example, Andersen et al. (1993)):

$$
\tilde{F}_{n}(y)=1-\prod_{i: Z_{(i)}<y}\left(\frac{n-i}{n-i+1}\right)^{\Delta_{(i)}},
$$

with the convention $0^{0}=1$. Here the $Z_{(i)}$ denote the ordered sequence of $Z_{i}$ and the $\Delta_{(i)}$ are corresponding indicators. A rich literature has been devoted to this estimator and its properties (see Andersen et al. (1993) and further references therein).

In the next theorem the local asymptotic performance of the estimator $\tilde{f}_{n}$ with respect to the topology $\tilde{\mathscr{T}}_{\delta}$ is established. The proofs of the theorems are given in Section 5.

Theorem 1. Let the distribution function $G$ be continuous at point $x$. Then the relation

$$
\limsup _{n \rightarrow \infty} \frac{n}{\log n} \mathrm{E}_{f}\left\{\tilde{f}_{n}(x)-f(x)\right\}^{2} \leqslant \sigma^{2}(f)
$$

holds locally uniformly in $f \in \tilde{\mathscr{F}}_{\delta}$, where

$$
\sigma^{2}(f)=\sigma^{2}(f, x)=\frac{f(x)}{2 \pi \delta\{1-G(x)\}}
$$

and the estimator $\tilde{f}_{n}(x)$ is defined by $(10)$.

Theorem 1 gives an upper bound for the local minimax risk (2); for a sufficiently small neighbourhood $V(f)$

$$
\limsup _{n \rightarrow \infty} \frac{n}{\log n} r_{n}(V) \leqslant \sup _{f \in V} \sigma(f) .
$$

If we can provide a lower bound for the local minimax risk, coinciding asymptotically with the upper one, then we clearly determine the asymptotic behaviour of the local minimax risk. The treatment of the lower bound is similar to that in Golubev and Levit (1996), with the difference that one has to take into account the censoring mechanism. The next theorem describes the lower bound for the local minimax risk.

Theorem 2. Let the distribution function $G$ be continuous at point $x$. Then, for each neighbourhood $V \subseteq \tilde{\mathscr{F}}_{\delta}$,

$$
\liminf _{n \rightarrow \infty} \frac{n}{\log n} r_{n}(V) \geqslant \sup _{f \in V} \sigma^{2}(f),
$$

where the local minimax risk $r_{n}(V)$ and $\sigma^{2}(f)$ are defined by (2) and (12), respectively.

In view of Theorems 1 and 2 , the estimator $\tilde{f}_{n}$ is efficient. Indeed, for each $f \in \tilde{\mathscr{F}}_{\delta}$ and for any sufficiently small neighbourhood $V(f)$,

$$
\lim _{n \rightarrow \infty} \frac{n}{\log n}\left(\sup _{f \in V} \mathrm{E}_{f}\left\{\tilde{f}_{n}(x)-f(x)\right\}^{2}-r_{n}(V)\right)=0 .
$$


Moreover, as an immediate consequence of Theorem 1 and Theorem 2, we obtain the asymptotic behaviour of the local minimax risk.

Corollary 1. Let $f \in \tilde{\mathscr{F}}_{\delta}$ and let the distribution function $G$ be continuous at point $x$. Then, for any sufficiently small neighbourhood $V(f)$,

$$
\lim _{n \rightarrow \infty} \frac{n}{\log n} r_{n}(V)=\sup _{f \in V} \sigma^{2}(f) .
$$

Since $\sigma^{2}(\cdot)$ is a continuous functional, this implies also that

$$
\lim _{V \downarrow f_{0}} \lim _{n \rightarrow \infty} \frac{n}{\log n} r_{n}(V)=\lim _{V \downarrow f_{0}} \sup _{f \in V} \sigma^{2}(f)=\sigma^{2}\left(f_{0}\right) .
$$

Remark 5. Note that, the smaller $\alpha$ and the larger $P, m, \tau_{0}$ in the definition of the class $\tilde{\mathscr{F}}_{\delta}$, the less restrictive is this class, while the asymptotic behaviour of the local minimax risk in no way depends on $\alpha, P, m$ and $\tau_{0}$.

Remark 6. Compared with the result of Golubev and Levit (1996), we see that the fact of censorship does not influence the convergence rate, but it does influence the optimal constant.

Remark 7. Since the Kaplan-Meier estimator is asymptotically normal, it seems plausible that a central limit theorem for the estimator $\tilde{f}_{n}(x)$ can be given (cf. Yang (1994)):

$$
\left(\frac{n}{\log n}\right)^{1 / 2}\left\{\tilde{f}_{n}(x)-f(x)\right\} \stackrel{D}{\rightarrow} \mathscr{N}\left(0, \sigma^{2}(f, x)\right) \text { as } n \rightarrow \infty,
$$

where $\sigma^{2}(f, x)$ is defined by (12). For related result, see Yang (1994) where a central limit theorem for the functional $\int \psi \mathrm{d} \tilde{F}_{n}$ is established; however, one cannot apply the methods of Yang (1994) to our functional $\tilde{f}_{n}(x)$ directly because of dependence of the kernel $\phi_{n}$ on $n$. This problem will not be treated here.

\section{Preliminaries: the Kaplan-Meier estimator}

Our treatment of the upper bound for the minimax risk relies heavily on the martingale approach to the Kaplan-Meier estimator (Gill 1980). Below we present necessary preliminaries, beginning with a suitable adaptation from Gill (1980).

Let $N_{n}$ be the process counting observed $X_{i}$, and $Y$ be the process giving the number at risk:

$$
\begin{gathered}
N_{n}(u)=\#\left\{i: Z_{i} \leqslant u, \Delta_{i}=1\right\}, \\
Y_{n}(u)=\#\left\{i: Z_{i} \geqslant u\right\}, \\
J_{n}(u)=I\left\{Y_{n}(u)>0\right\},
\end{gathered}
$$


where the symbol \# denotes the number of elements in a set. Let $X(u-)$ denote left-hand limit of $X$ at point $u$. It is known (see, for example, Gill (1980)) that, for $y$ such that $F(y)<1$ and $Y_{n}(y)>0$,

$$
\begin{aligned}
\tilde{F}_{n}(y)-F(y) & =\{1-F(y)\} \int_{-\infty}^{y} \frac{\left\{1-\tilde{F}_{n}(u-)\right\}}{\{1-F(u)\}} \frac{J_{n}(u)}{Y_{n}(u)}\left(\mathrm{d} N_{n}(u)-Y_{n}(u) \frac{\mathrm{d} F(u)}{1-F(u-)}\right) \\
& =\{1-F(y)\} \int_{-\infty}^{y} \frac{\left\{1-\tilde{F}_{n}(u-)\right\}}{\{1-F(u)\}} \frac{J_{n}(u)}{Y_{n}(u)} \mathrm{d} M_{n}(u),
\end{aligned}
$$

where $M_{n}(u)$ is a square integrable martingale with the predictable variation process

$$
\langle M, M\rangle(y)=\int_{-\infty}^{y} Y_{n}(u) \frac{1-F(u)}{\{1-F(u-)\}^{2}} \mathrm{~d} F(u),
$$

while $J_{n}(u), \tilde{F}_{n}(u-), Y_{n}(u)$ are left continuous adapted processes.

We close this section with two technical lemmas which will be needed in the following section.

For the following result we refer to Weits (1993).

Lemma 1. Let $\mathscr{B}=\mathscr{B}(A, \tau)=\{F:\{1-F(A)\}\{1-G(A)\} \geqslant \tau>0\}$. Then, as $n \rightarrow \infty$, the relation

$$
\mathrm{E} \frac{\left\{1-\tilde{F}_{n}(y-)\right\}^{2}}{\{1-F(y-)\}^{2}} \frac{J_{n}(y)}{Y_{n}(y)}=\frac{1}{n\{1-F(y-)\}\{1-G(y-)\}}+O\left(n^{-2}\right)
$$

holds uniformly over $\mathscr{B}$ and $y, y \leqslant A$.

The proof of this lemma is essentially contained in the proof of Lemma 4 in Weits (1993). In the paper of Weits, $Y_{n} / n$ corresponds to our $Y_{n}$.

Lemma 2. For all $n \geqslant 2$,

$$
\mathrm{E}\left(\frac{J_{n}(y)}{Y_{n}(y)}\right) \leqslant p(y)\{1-p(y)\}^{n-1}+\frac{\{1-p(y)\}^{n / 2}}{p(y)}+\frac{2}{n p(y)},
$$

where $p=p(y)=\{1-F(y-)\}\{1-G(y-)\}$.

Proof. Denote

$$
\mu(n)=\mu(n, y)=n \mathrm{E}\left(\frac{J_{n}(y)}{Y_{n}(y)}\right)=\sum_{l=1}^{n} \frac{1}{l / n}\left(\begin{array}{c}
n \\
l
\end{array}\right) p^{l}(1-p)^{n-l}
$$

Reasoning as in Weits (1993), we obtain the following recursive equation for $\mu(n)$ : 


$$
\begin{aligned}
\mu(n) & =n \sum_{l=1}^{n-1} \frac{1}{l}\left\{\left(\begin{array}{c}
n-1 \\
l-1
\end{array}\right)+\left(\begin{array}{c}
n-1 \\
l
\end{array}\right)\right\} p^{l}(1-p)^{n-l}+p^{n} \\
& =n\left\{\frac{1}{n} \sum_{l=1}^{n} \frac{n !}{(n-l) ! l !} p^{l}(1-p)^{n-l}+(1-p) \sum_{l=1}^{n-1} \frac{1}{l}\left(\begin{array}{c}
n-1 \\
l
\end{array}\right) p^{l}(1-p)^{n-l-1}\right\} \\
& =n\left(\frac{1}{n}\left\{1-(1-p)^{n}\right\}+(1-p) \frac{1}{n-1} \mu(n-1)\right) \\
& =1+(1-p) \frac{n}{n-1} \mu(n-1)-(1-p)^{n} .
\end{aligned}
$$

Certainly $\mu(n) \leqslant \lambda(n)$, where $\lambda(n)$ satisfies the recursive equation

$$
\lambda(n)=1+(1-p) \frac{n}{n-1} \lambda(n-1), \quad \lambda(1)=\mu(1)=p, \quad n \geqslant 2 .
$$

Let $C(n)$ be a solution of the corresponding homogeneous equation:

$$
C(n)=(1-p) \frac{n}{n-1} C(n-1) \quad C(1)=1, \quad n \geqslant 2 .
$$

Thus,

$$
C(n)=(1-p)^{n-1} n .
$$

Let $B(n)$ be such that $\lambda(n)=C(n) B(n)$. Then $B(n)$ satisfies

$$
B(n)=B(n-1)+\frac{n-1}{n(1-p)}\{C(n-1)\}^{-1}, \quad B(1)=p, \quad n \geqslant 2 .
$$

It is easy to see that

$$
B(n)=p+\sum_{k=2}^{n}(1-p)^{1-k} k^{-1}
$$

and

$$
\lambda(n)=p(1-p)^{n-1} n+n \sum_{k=2}^{n}(1-p)^{n-k} k^{-1} .
$$

Denote by $\lfloor C\rfloor$ the whole part of $C$.

Now we bound the second term in the last relation

$$
\begin{aligned}
\sum_{k=2}^{n}(1-p)^{n-k} k^{-1} & \leqslant \sum_{k=1}^{\lfloor n / 2\rfloor}(1-p)^{n-k}+\frac{1}{\lfloor n / 2\rfloor+1} \sum_{k=\lfloor n / 2\rfloor+1}^{n}(1-p)^{n-k} \\
& \leqslant \frac{(1-p)^{n / 2}}{p}+\frac{2}{n p}
\end{aligned}
$$


and hence the claim follows:

$$
\mathrm{E}\left(\frac{J_{n}(y)}{Y_{n}(y)}\right)=\frac{\mu(n)}{n} \leqslant \frac{\lambda(n)}{n} \leqslant p(1-p)^{n-1}+\frac{(1-p)^{n / 2}}{p}+\frac{2}{n p} .
$$

\section{Auxiliary results}

In this section we provide further technical results which we shall need in the proof of theorems.

Lemma 3. Let $\phi_{n}(y)$ be defined by (5) and $h(y)$ be bounded and continuous at the $x$ function. Then, as $n \rightarrow \infty$, the relation

$$
\int \phi_{n}^{2}(x-y) h(y) \mathrm{d} F(y)=h(x) f(x) \int \phi_{n}^{2}(y) \mathrm{d} y\{1+o(1)\}=\frac{h(x) f(x) \log n}{2 \pi \delta}\{1+o(1)\}
$$

holds locally uniformly in $F \in \mathscr{F}_{\delta}$,

Proof. The second relation follows immediately from (8). It remains to prove only the first identity. Let $O_{\epsilon}(x)=\{y:|x-y|<\epsilon\}$ be the open interval around $x$ radius $\epsilon=\epsilon_{n}, \epsilon_{n} \rightarrow 0$, $\left(\epsilon_{n}^{2} \log n\right)^{-1}=o(1)$ as $n \rightarrow \infty$. We have obviously

$$
\begin{aligned}
\int \phi_{n}^{2}(x-y) h(y) \mathrm{d} F(y)-h(x) f(x) \int \phi_{n}^{2}(y) \mathrm{d} y= & \int_{O_{\epsilon}(x)} \phi_{n}^{2}(x-y)\{h(y) f(y)-h(x) f(x)\} \mathrm{d} y \\
& +\int_{\left\{O_{\epsilon}(x)\right\}^{C}} \phi_{n}^{2}(x-y)\{h(y) f(y)-h(x) f(x)\} \mathrm{d} y .
\end{aligned}
$$

So it is enough to prove that the right-hand side of the last identity is of order $o(\log n)$ locally uniformly.

One can bound the function $\phi_{n}(x-y)$ outside the interval $O_{\epsilon}(x)$ as follows:

$$
\phi_{n}^{2}(x-y) \leqslant \frac{\mathrm{e}^{-2 \tau(x-y)^{2 m}}}{\pi^{2} \epsilon_{n}^{2}} .
$$

Therefore, the inequality

$$
\int_{\left\{O_{\epsilon}(x)\right\}^{C}} \phi_{n}^{2}(x-y)|h(y) f(y)-h(x) f(x)| \mathrm{d} y \leqslant C_{1} \epsilon_{n}^{-2} \int_{\left(O_{\epsilon}(x)\right)^{C}} \mathrm{e}^{-2 \tau(x-y)^{2 m}} \mathrm{~d} y=o(\log n)
$$

holds locally uniformly because our topology is stronger than the topology induced by the sup norm (see Remark 2). Next, by (8), it is easy to see that 


$$
\int_{O_{\epsilon}(x)} \phi_{n}^{2}(x-y)\{h(y) f(y)-h(x) f(x)\} \mathrm{d} y=o(1) \int \phi_{n}^{2}(y) \mathrm{d} y=o(\log n)
$$

locally uniformly and the lemma follows.

Lemma 4. The functional

$$
\psi_{n}(y)=\psi_{n}(y, x, F)=\int_{y}^{\infty} \phi_{n}(x-u) \mathrm{d} F(u)
$$

is bounded locally uniformly in $F \in \tilde{\mathscr{F}}_{\delta}$ and uniformly in $y$.

Proof. Denote $D_{1}(y)=O_{\epsilon}(x) \cap[y,+\infty)=\left(b_{1}, b_{2}\right), \quad D_{2}(y)=\left\{O_{\epsilon}(x)\right\}^{C} \cap[y,+\infty)$, where $O_{\epsilon}(x)$ is the open interval around $x$ of radius $\epsilon$. Then

$$
\psi_{n}(y)=\int_{D_{1}(y)} \phi_{n}(x-u) \mathrm{d} F(u)+\int_{D_{2}(y)} \phi_{n}(x-u) \mathrm{d} F(u) .
$$

The second term (integral over $D_{2}(y)$ ) is clearly bounded. For the first term, we have that

$$
\begin{aligned}
\left|\int_{D_{1}(y)} \phi_{n}(x-u) \mathrm{d} F(u)\right| \leqslant & \left|f(x) \int_{D_{1}(y)} \phi_{n}(x-u) \mathrm{d} u\right| \\
& +\left|\int_{D_{1}(y)} f^{\prime}\left(u^{*}\right)(u-x) \phi_{n}(x-u) \mathrm{d} u\right| \\
& \leqslant C_{1}+C_{2} \int_{D_{1}(y)} \mathrm{e}^{-\tau(x-u)^{2 m}} \mathrm{~d} u \\
& \leqslant C_{3}
\end{aligned}
$$

locally uniformly because $\sup _{u \in O_{\epsilon}(x)}\left|f^{\prime}(u)\right|$ is bounded locally uniformly (see Remark 2) and

$$
\left|\int_{D_{1}(y)} \phi_{n}(x-u) \mathrm{d} u\right|=\left|\int_{a_{n}\left(b_{1}-x\right)}^{a_{n}\left(b_{2}-x\right)} \frac{\mathrm{e}^{-\tau\left(u / a_{n}\right)^{2 m}} \sin u}{\pi u} \mathrm{~d} u\right| \leqslant C_{4} .
$$

Lemma 5. As $n \rightarrow \infty$, the relation

$$
b_{n}^{2}(x)=\left(\int \phi_{n}(x-y) \mathrm{d} F(y)-f(x)\right)^{2}=O\left(n^{-1}\right)
$$

holds uniformly over $\tilde{\mathscr{F}}_{\delta}$.

Proof. Recalling the definition of the class (4), we obtain the following uniform bound: 


$$
\begin{aligned}
\left(\int \phi_{n}(x-y) \mathrm{d} F(y)-f(x)\right)^{2} & =\left(\frac{1}{2 \pi} \int \mathrm{e}^{-\mathrm{i} t x}\left\{\hat{\phi}_{n}(t)-1\right\} \hat{f}(t) \mathrm{d} t\right)^{2} \\
& \leqslant \frac{1}{2 \pi} \int \cosh ^{2}(\delta t)|\hat{f}(t)|^{2} \mathrm{~d} t \frac{1}{2 \pi} \int \frac{\left|\hat{\phi}_{n}(t)-1\right|^{2}}{\{\cosh (\delta t)\}^{2}} \mathrm{~d} t \\
& \leqslant C_{1} \int \frac{\left|\hat{\phi}_{n}(t)-1\right|^{2}}{\{\cosh (\delta t)\}^{2}} \mathrm{~d} t \\
& \leqslant C_{1} \int_{-a_{n}}^{a_{n}} \frac{\left|\hat{\phi}_{n}(t)-1\right|^{2}}{\{\cosh (\delta t)\}^{2}} \mathrm{~d} t+C_{2} \int_{|t| \geqslant a_{n}} \frac{\mathrm{d} t}{\{\cosh (\delta t)\}^{2}} \\
& =2 C_{1} \int_{0}^{a_{n}} \frac{\left|\hat{\phi}_{n}(t)-1\right|^{2}}{\{\cosh (\delta t)\}^{2}} \mathrm{~d} t+O\left(n^{-1}\right) .
\end{aligned}
$$

Since the function $\hat{r}(u)$ is even,

$$
\int_{u>t+a_{n}}|\hat{r}(u)| \mathrm{d} u \leqslant \int_{u<t-a_{n}}|\hat{r}(u)| \mathrm{d} u=\int_{u>a_{n}-t}|\hat{r}(u)| \mathrm{d} u
$$

for $t \in\left[0, a_{n}\right]$. Now using the last inequality, (7), (9) and the fact that

$$
\int \hat{r}(u) \mathrm{d} u=r(0) 2 \pi=2 \pi
$$

we have that, for $t \in\left[0, a_{n}\right]$,

$$
\begin{aligned}
\left|\hat{\phi}_{n}(t)-1\right| & =\left|(2 \pi)^{-1}\left(\hat{r} * I_{\left(-a_{n}, a_{n}\right)}\right)(t)-1\right| \\
& \left.=\mid(2 \pi)^{-1} \int\left(I_{\left(-a_{n}, a_{n}\right)}\right)(t-u)-1\right) \hat{r}(u) \mathrm{d} u \mid \\
& =(2 \pi)^{-1}\left|\int_{|t-u|>a_{n}} \hat{r}(u) \mathrm{d} u\right| \\
& \leqslant 2(2 \pi)^{-1} \int_{u>a_{n}-t}|\hat{r}(u)| \mathrm{d} u \\
& \leqslant C_{3} \int_{u>a_{n}-t} \exp \left(-A_{2} u^{2 m /(2 m-1)}\right) \mathrm{d} u \\
& \leqslant C_{4} \exp \left\{-A_{2}\left(a_{n}-t\right)^{2 m /(2 m-1)}\right\} .
\end{aligned}
$$


Combining this with (16) completes the proof:

$$
\begin{aligned}
\int_{0}^{a_{n}} \frac{\left|\hat{\phi}_{n}(t)-1\right|^{2}}{\{\cosh (\delta t)\}^{2}} \mathrm{~d} t & \leqslant C_{5} \int_{0}^{a_{n}} \exp \left\{-2 A_{2}\left(a_{n}-t\right)^{2 m /(2 m-1)}-2 \delta t\right\} \mathrm{d} t \\
& =C_{5} \int_{0}^{a_{n}} \exp \left\{-2 A_{2} y^{2 m /(2 m-1)}-2 \delta\left(a_{n}-y\right)\right\} \mathrm{d} y \\
& \leqslant C_{5} \mathrm{e}^{-2 \delta a_{n}} \int_{0}^{\infty} \exp \left(-2 A_{2} y^{2 m /(2 m-1)}+2 \delta y\right) \mathrm{d} y \\
& =\frac{C_{6}}{n}
\end{aligned}
$$

Lemma 6. As $n \rightarrow \infty$, the relations

$$
\begin{aligned}
\mathrm{E}\left(\frac{\psi_{n}\left(Z_{(n)}\right)}{1-F\left(Z_{(n)}\right)}\right)^{2} & =O\left(n^{-1}\right), \\
\mathrm{E}\left(\psi_{n}\left(Z_{(n)}\right)\right)^{2} & =O\left(n^{-1}\right),
\end{aligned}
$$

hold locally uniformly in $F \in \tilde{\mathscr{F}}_{\delta}$, where $\psi_{n}(y)$ is defined by (15).

Proof. From the definition of the nonparametric class (4) it follows that, for each $F \in \tilde{\mathscr{F}}_{\delta}$,

$$
H(y) \stackrel{\text { def }}{=} \mathbf{P}\left\{Z_{1} \leqslant y\right\}=1-\{1-F(y)\}\{1-G(y)\} \leqslant 1-\alpha \mathrm{e}^{-\tau_{0} y^{2 m}} .
$$

Fix some $\epsilon>0$ and note that

$$
H(y) \leqslant H(x+\epsilon) \leqslant 1-\alpha \mathrm{e}^{-\tau_{0}(x+\epsilon)^{2 m}}=q<1 \quad \text { for all } y \leqslant x+\epsilon .
$$

Further, by the definition of the kernel function (5), it is easy to see that, with some constant $C_{1}>0$,

$$
\phi_{n}^{2}(x-y) \leqslant(\pi \epsilon)^{-2} \mathrm{e}^{-2 \tau(x-y)^{2 m}} \leqslant C_{1} \mathrm{e}^{-3 \tau_{0} y^{2 m}} \quad \text { for all } y \geqslant x+\epsilon
$$

Also, we have obviously that

$$
\phi_{n}^{2}(y) \leqslant a_{n}^{2} \pi^{-2}
$$

Now, using the Hölder inequality and all the inequalities above, we obtain the second relation 


$$
\begin{aligned}
\mathrm{E}\left(\int_{Z_{(n)}}^{\infty} \phi_{n}(x-y) \mathrm{d} F(y)\right)^{2} & \leqslant \mathrm{E} \int_{-\infty}^{\infty} I\left\{Z_{(n)} \leqslant y\right\} \phi_{n}^{2}(x-y) \mathrm{d} F(y) \\
& =\int_{-\infty}^{\infty} H^{n}(y) \phi_{n}^{2}(x-y) \mathrm{d} F(y) \\
& =\int_{-\infty}^{x+\epsilon} H^{n}(y) \phi_{n}^{2}(x-y) \mathrm{d} F(y)+\int_{x+\epsilon}^{\infty} H^{n}(y) \phi_{n}^{2}(x-y) \mathrm{d} F(y) \\
& \leqslant q^{n} \pi^{-2} a_{n}^{2} F(x+\epsilon)+C_{2} \int_{x+\epsilon}^{\infty}\left(1-\alpha \mathrm{e}^{-\tau_{0} y^{2 m}}\right)^{n} \phi_{n}^{2}(x-y) \mathrm{d} y \\
& \leqslant q^{n} \pi^{-2} a_{n}^{2}+C_{3} \int_{x+\epsilon}^{\infty} \mathrm{e}^{-3 \tau_{0} y^{2 m}}\left(1-\alpha \mathrm{e}^{-\tau_{0} y^{2 m}}\right)^{n} \mathrm{~d} y \\
& \leqslant C_{4} \mathrm{e}^{-C_{5} n}(\log n)^{2}+C_{6} \int_{0}^{C_{7}}(1-u)^{n} \mathrm{~d} u \\
& =O\left(n^{-1}\right)
\end{aligned}
$$

locally uniformly because $f(y)$ is bounded locally uniformly and $0<C_{7} \leqslant 1$.

To prove the first relation, note that from (4)

$$
\begin{aligned}
\mathrm{E}\left(\frac{\psi_{n}\left(Z_{(n)}\right)}{1-F\left(Z_{(n)}\right)}\right)^{2} & =\mathrm{E}\left(\frac{\psi_{n}\left(Z_{(n)}\right)}{1-F\left(Z_{(n)}\right)}\left(I\left\{Z_{(n)} \leqslant x+\epsilon\right\}+I\left\{Z_{(n)}>x+\epsilon\right\}\right)\right)^{2} \\
& \leqslant C_{8} \mathrm{E}\left\{\psi_{n}\left(Z_{(n)}\right)\right\}^{2}+2 \alpha^{-2} \mathrm{E}\left[\exp \left\{2 \tau_{0}\left(Z_{(n)}\right)^{2 m}\right\} I\left\{Z_{(n)}>x+\epsilon\right\} \psi_{n}^{2}\left(Z_{(n)}\right)\right]
\end{aligned}
$$

Therefore, it remains only to show that the second term in the right-hand side of the last inequality is of order $O\left(n^{-1}\right)$ locally uniformly:

$$
\begin{gathered}
\mathrm{E}\left[\exp \left\{2 \tau_{0}\left(Z_{(n)}\right)^{2 m}\right\} I\left\{Z_{(n)}>x+\epsilon\right\} \psi_{n}^{2}\left(Z_{(n)}\right)\right] \\
=\mathrm{E}\left\{\exp \left\{2 \tau_{0}\left(Z_{(n)}\right)^{2 m}\right\} I\left\{Z_{(n)}>x+\epsilon\right\}\left(\int_{Z_{(n)}}^{\infty} \phi_{n}(x-y) \mathrm{d} F(y)\right)^{2}\right\} \\
\leqslant \mathrm{E}\left(\exp \left\{2 \tau_{0}\left(Z_{(n)}\right)^{2 m}\right\} I\left\{Z_{(n)}>x+\epsilon\right\} \int_{Z_{(n)}}^{\infty} \phi_{n}^{2}(x-y) \mathrm{d} F(y)\right) \\
\leqslant C_{9} \mathrm{E}\left(\exp \left\{2 \tau_{0}\left(Z_{(n)}\right)^{2 m}\right\} I\left\{Z_{(n)}>x+\epsilon\right\} \int_{Z_{(n)}}^{\infty} \mathrm{e}^{-3 \tau_{0} y^{2 m}} \mathrm{~d} y\right)
\end{gathered}
$$




$$
\begin{aligned}
& \leqslant C_{9} \mathrm{E}\left(\int_{Z_{(n)}}^{\infty} \mathrm{e}^{-\tau_{0} y^{2 m}} \mathrm{~d} y\right) \\
& =C_{9} \int H^{n}(y) \mathrm{e}^{-\tau_{0} y^{2 m}} \mathrm{~d} y \\
& \leqslant C_{9} \int\left(1-\alpha \mathrm{e}^{-\tau_{0} y^{2 m}}\right)^{n} \mathrm{e}^{-\tau_{0} y^{2 m}} \mathrm{~d} y \\
& \leqslant C_{10} \int_{0}^{1}(1-u)^{n} \mathrm{~d} u \\
& =O\left(n^{-1}\right)
\end{aligned}
$$

locally uniformly.

For brevity, denote from now on

$$
\bar{F}(y)=1-F(y) .
$$

Lemma 7. Let the distribution function $G$ be continuous at the point $x$. Then, as $n \rightarrow \infty$, the relation

$$
\begin{array}{r}
\frac{n}{\log n} \mathrm{E}\left(\int_{-\infty}^{Z_{(n)}}\left\{\phi_{n}(x-y) \bar{F}(y)-\psi_{n}(y)\right\}^{2} \frac{\left\{1-\tilde{F}_{n}(y-)\right\}^{2}}{\{1-F(y-)\}^{4}} \frac{J_{n}(y)}{Y_{n}(y)} \bar{F}(y) \mathrm{d} F(y)\right) \\
=\sigma^{2}(f)\{1+o(1)\}
\end{array}
$$

holds locally uniformly in $F \in \tilde{\mathscr{F}}_{\delta}$, where $\sigma^{2}(f)$ and $\psi_{n}$ are defined by (12) and (15), respectively.

Proof. By continuity of $F(y)$, we write the left-hand side of (19), for some positive $\epsilon$, as a sum of two terms:

$$
I_{1}=\mathrm{E}\left(\int_{-\infty}^{Z_{(n)}}\left\{\phi_{n}(x-y) \bar{F}(y)-\psi_{n}(y)\right\}^{2} \frac{\left\{1-\tilde{F}_{n}(y-)\right\}^{2}}{\{1-F(y-)\}^{3}} \frac{J_{n}(y)}{Y_{n}(y)} I\left\{Z_{(n)} \leqslant x+\epsilon\right\} \mathrm{d} F(y)\right)
$$

and

$$
I_{2}=\mathrm{E}\left(\int_{-\infty}^{Z_{(n)}}\left\{\phi_{n}(x-y) \bar{F}(y)-\psi_{n}(y)\right\}^{2} \frac{\left\{1-\tilde{F}_{n}(y-)\right\}^{2}}{\{1-F(y-)\}^{3}} \frac{J_{n}(y)}{Y_{n}(y)} I\left\{Z_{(n)}>x+\epsilon\right\} \mathrm{d} F(y)\right)
$$

To evaluate the first term, observe first that by (18) 


$$
\begin{aligned}
\mathbf{P}\left\{Z_{1} \leqslant x+\epsilon\right\}=H(x+\epsilon) & \leqslant 1-\alpha \mathrm{e}^{-\tau_{0}(x+\epsilon)^{2 m}}=q<1, \\
\left\{\phi_{n}(x-y) \bar{F}(y)-\psi_{n}(y)\right\}^{2} & \leqslant 2 \phi_{n}^{2}(x-y) \bar{F}^{2}(y)+2 \psi_{n}^{2}(y) \\
& \leqslant 4 \pi^{-2} a_{n}^{2} \mathrm{e}^{-2 \tau(x-y)^{2 m}} \bar{F}^{2}(y) \\
& =C_{1}(\log n)^{2} \mathrm{e}^{-2 \tau(x-y)^{2 m}} \bar{F}^{2}(y) \\
& \leqslant C_{2}(\log n)^{2} \mathrm{e}^{-3 \tau_{0} y^{2 m}} \bar{F}^{2}(y)
\end{aligned}
$$

and $J_{n}(y) / Y_{n}(y) \leqslant 1$. Thus, recalling (4), we bound the first term as follows:

$$
\begin{aligned}
I_{1} & \leqslant C_{2}(\log n)^{2} H^{n}(x+\epsilon) \int_{-\infty}^{\infty} \frac{\mathrm{e}^{-3 \tau_{0} y^{2 m}} \mathrm{~d} F(y)}{1-F(y)} \\
& \leqslant C_{2}(\log n)^{2} q^{n} \alpha^{-1} \int_{-\infty}^{\infty} \mathrm{e}^{-3 \tau_{0} y^{2 m}+\tau_{0} y^{2 m}} \mathrm{~d} F(y) \\
& \leqslant C_{3} \mathrm{e}^{-C_{4} n} .
\end{aligned}
$$

For the second term, we split the integral in $I_{2}$ into two parts: the integral over $(-\infty, x+\epsilon]$ and the integral over $\left(x+\epsilon, Z_{(n)}\right]$. Since, for $y \geqslant x+\epsilon$,

$$
\begin{aligned}
\left\{\phi_{n}(x-y) \bar{F}(y)-\psi_{n}(y)\right\}^{2} & \leqslant 2 \phi_{n}^{2}(x-y) \bar{F}^{2}(y)+2 \psi_{n}^{2}(y) \\
& \leqslant 4(\pi \epsilon)^{-2} \mathrm{e}^{-2 \tau(x-y)^{2 m}} \bar{F}^{2}(y) \\
& \leqslant C_{5} \mathrm{e}^{-3 \tau_{0} y^{2 m}} \bar{F}^{2}(y),
\end{aligned}
$$

we bound the expectation of the integral over $\left(x+\epsilon, Z_{(n)}\right]$ merely by

$$
C_{5} \int_{x+\epsilon}^{\infty} \mathrm{E}\left(\frac{J_{n}(y)}{Y_{n}(y)}\right) \frac{\mathrm{e}^{-3 \tau_{0} y^{2 m}} \mathrm{~d} F(y)}{1-F(y)} .
$$

Thus,

$$
\begin{aligned}
I_{2} \leqslant & \mathrm{E}\left(\int_{-\infty}^{x+\epsilon}\left\{\phi_{n}(x-y) \bar{F}(y)-\psi_{n}(y)\right\}^{2} \frac{\left\{1-\tilde{F}_{n}(y-)\right\}^{2}}{\{1-F(y-)\}^{3}} \frac{J_{n}(y)}{Y_{n}(y)} \mathrm{d} F(y)\right) \\
& +C_{5} \int_{x+\epsilon}^{\infty} \mathrm{E}\left(\frac{J_{n}(y)}{Y_{n}(y)}\right) \frac{\mathrm{e}^{-3 \tau_{0} y^{2 m}} \mathrm{~d} F(y)}{1-F(y)} \\
= & S_{1}+S_{2},
\end{aligned}
$$

say. 
To evaluate $S_{2}$, we make use of Lemma 2 and (4):

$$
\begin{aligned}
S_{2} & =C_{5} \int_{x+\epsilon}^{\infty} \mathrm{e}^{-3 \tau_{0} y^{2 m}} \mathrm{E}\left(\frac{J_{n}(y)}{Y_{n}(y)}\right) \frac{\mathrm{d} F(y)}{1-F(y)} \\
& \leqslant 2 C_{5} \int_{x+\epsilon}^{\infty} \frac{\mathrm{e}^{-3 \tau_{0} y^{2 m}}\left\{H^{n / 2}(y)+n^{-1}\right\} \mathrm{d} F(y)}{\{1-F(y)\}^{2}\{1-G(y)\}} \\
& \left.\leqslant C_{6} \int_{x+\epsilon}^{\infty} \mathrm{e}^{-3 \tau_{0} y^{2 m}+2 \tau_{0} y^{2 m}}\left\{\left(1-\alpha \mathrm{e}^{-\tau_{0} y^{2 m}}\right)^{n / 2}+n^{-1}\right)\right\} f(y) \mathrm{d} y \\
& \leqslant C_{7} \int_{0}^{C_{8}}(1-u)^{n / 2} \mathrm{~d} u+C_{6} n^{-1} \\
& =O\left(n^{-1}\right)
\end{aligned}
$$

locally uniformly because $0<C_{8} \leqslant 1$.

Therefore, to complete the proof, it remains only to prove that the relation

$$
\frac{n}{\log n} S_{1}=\sigma^{2}(f)\{1+o(1)\}
$$

holds locally uniformly.

Since

$$
\{1-F(x+\epsilon)\}\{1-G(x+\epsilon)\} \geqslant \alpha \mathrm{e}^{-\tau_{0}(x+\epsilon)^{2 m}}=\gamma>0
$$

uniformly over $\tilde{\mathscr{F}}_{\delta}$, by (20) and Lemma 1 we have that

$$
\begin{aligned}
S_{1} & =\mathrm{E}\left(\int_{-\infty}^{x+\epsilon}\left\{\phi_{n}(x-y) \bar{F}(y)-\psi_{n}(y)\right\}^{2} \frac{\left\{1-\tilde{F}_{n}(y-)\right\}^{2}}{\{1-F(y-)\}^{3}} \frac{J_{n}(y)}{Y_{n}(y)} \mathrm{d} F(y)\right) \\
& =\int_{-\infty}^{x+\epsilon} \frac{\left\{\phi_{n}(x-y) \bar{F}(y)-\psi_{n}(y)\right\}^{2} \mathrm{~d} F(y)}{n\{1-F(y)\}^{2}\{1-G(y-)\}}+O\left\{(\log n)^{2} n^{-2}\right\}
\end{aligned}
$$

uniformly. Finally, we obtain by Lemmas 3 and 4 that

$$
S_{1}=\int_{-\infty}^{x+\epsilon} \frac{\phi_{n}^{2}(x-y) \mathrm{d} F(y)}{n\{1-G(y-)\}}+O\left(n^{-1}\right)=\frac{\log n}{n} \sigma^{2}(f)\{1+o(1)\}
$$

locally uniformly. Thus (22) holds and the proof of Lemma 7 is complete.

\section{Proofs of theorems}

Upper bound: proof of Theorem 1. First we provide the necessary preliminaries. By (13) and integration by parts, we have 


$$
\begin{aligned}
\int_{-\infty}^{Z_{(n)}} \phi_{n}(x-y) \mathrm{d}\left\{\tilde{F}_{n}(y)-F(y)\right\}= & \int_{-\infty}^{Z_{(n)}}\left\{\phi_{n}(x-y) \bar{F}(y)-\psi_{n}(y)\right\} \frac{1-\tilde{F}_{n}(y-)}{1-F(y-)} \frac{J_{n}(y)}{Y_{n}(y)} \mathrm{d} M_{n}(y) \\
& +\frac{\tilde{F}_{n}\left(Z_{(n)}\right)-F\left(Z_{(n)}\right)}{1-F\left(Z_{(n)}\right)} \psi_{n}\left(Z_{(n)}\right) .
\end{aligned}
$$

Since the first term of (23) is the integral of a predictable locally bounded process (almost all its sample paths are locally bounded) with respect to a square integrable martingale with the predictable variation process (14) (see, for example, Gill (1980)), one can represent its second moment as follows:

$$
\begin{aligned}
& \mathrm{E}\left(\int_{-\infty}^{Z_{(n)}}\left\{\phi_{n}(x-y) \bar{F}(y)-\psi_{n}(y)\right\} \frac{1-\tilde{F}_{n}(y-)}{1-F(y-)} \frac{J_{n}(y)}{Y_{n}(y)} \mathrm{d} M_{n}(y)\right)^{2} \\
= & \mathrm{E} \int_{-\infty}^{Z_{(n)}}\left\{\phi_{n}(x-y) \bar{F}(y)-\psi_{n}(y)\right\}^{2} \frac{\left\{1-\tilde{F}_{n}(y-)\right\}^{2}}{\{1-F(y-)\}^{4}} \frac{J_{n}(y)}{Y_{n}(y)} \bar{F}(y) \mathrm{d} F(y) .
\end{aligned}
$$

Recall that $\tilde{F}_{n}(y)$ is constant on $\left[Z_{(n)}, \infty\right)$. So, using (23), (24) and (twice) the elementary inequality

$$
(a+b)^{2} \leqslant(1+\gamma) a^{2}+\left(1+\gamma^{-1}\right) b^{2}, \quad 0<\gamma \leqslant 1,
$$

we obtain

$$
\begin{gathered}
\mathrm{E}\left(\int_{-\infty}^{\infty} \phi_{n}(x-y) \mathrm{d}\left\{\tilde{F}_{n}(y)-F(y)\right\}\right)^{2} \\
\leqslant\left(1+\gamma_{n}\right) \mathrm{E}\left(\int_{-\infty}^{Z_{(n)}} \phi_{n}(x-y) \mathrm{d}\left\{\tilde{F}_{n}(y)-F(y)\right\}\right)^{2}+\left(1+\gamma_{n}^{-1}\right) \mathrm{E}\left\{\psi_{n}\left(Z_{(n)}\right)\right\}^{2} \\
\leqslant\left(1+\gamma_{n}\right)^{2} \mathrm{E}\left(\int_{-\infty}^{Z_{(n)}}\left\{\phi_{n}(x-y) \bar{F}(y)-\psi_{n}(y)\right\}^{2} \frac{\left\{1-\tilde{F}_{n}(y-)\right\}^{2}}{\{1-F(y-)\}^{4}} \frac{J_{n}(y)}{Y_{n}(y)} \bar{F}(y) \mathrm{d} F(y)\right) \\
+\left(\gamma_{n}^{-1}+2+\gamma_{n}\right) \mathrm{E}\left(\frac{\psi_{n}\left(Z_{(n)}\right)}{1-F\left(Z_{(n)}\right)}\right)^{2}+\left(1+\gamma_{n}^{-1}\right) \mathrm{E}\left\{\psi_{n}\left(Z_{(n)}\right)\right\}^{2},
\end{gathered}
$$

where $\gamma_{n}$ is to be chosen later.

Now we evaluate the risk of the estimator (10). From the last inequality and again the elementary inequality (25) it follows that

$$
\begin{aligned}
\mathrm{E}_{f}\left(\tilde{f}_{n}(x)-f(x)\right)^{2} & =\mathrm{E}_{f}\left(\tilde{f}_{n}(x)-\int \phi_{n}(x-y) \mathrm{d} F(y)+\int \phi_{n}(x-y) \mathrm{d} F(y)-f(x)\right)^{2} \\
& \leqslant\left(1+\gamma_{n}\right) \mathrm{E}\left(\int \phi_{n}(x-y) \mathrm{d}\left\{\tilde{F}_{n}(y)-F(y)\right\}\right)^{2}
\end{aligned}
$$




$$
\begin{aligned}
& +\left(1+\gamma_{n}^{-1}\right)\left(\int \phi_{n}(x-y) \mathrm{d} F(y)-f(x)\right)^{2} \\
\leqslant & \left(1+\gamma_{n}\right)^{3} \mathrm{E} \int_{-\infty}^{Z_{(n)}}\left\{\phi_{n}(x-y) \bar{F}(y)-\psi_{n}(y)\right\}^{2} \frac{\left\{1-\tilde{F}_{n}(y-)\right\}^{2}}{\{1-F(y-)\}^{4}} \frac{J_{n}(y)}{Y_{n}(y)} \bar{F}(y) \mathrm{d} F(y) \\
& +\left(\gamma_{n}^{-1}+3+3 \gamma_{n}+\gamma_{n}^{2}\right) \mathrm{E}\left(\frac{\psi_{n}\left(Z_{(n)}\right)}{1-F\left(Z_{(n)}\right)}\right)^{2}+\left(\gamma_{n}^{-1}+2+\gamma_{n}\right) \mathrm{E}\left\{\psi_{n}\left(Z_{(n)}\right)\right\}^{2} \\
& +\left(1+\gamma_{n}^{-1}\right)\left(\int \phi_{n}(x-y) \mathrm{d} F(y)-f(x)\right)^{2}
\end{aligned}
$$

We choose now $\gamma_{n}$ such that $\gamma_{n} \rightarrow 0$ and $\left(\gamma_{n} \log n\right)^{-1}=o(1)$ as $n \rightarrow \infty$. Combining the last relation with Lemmas 5-7 proves the theorem.

Lower bound: proof of Theorem 2. Let $f_{0}(y)$ be an arbitrary density from neighbourhood $V$ and let $F_{0}$ be the corresponding distribution function. Consider the following family of functions (cf. Golubev and Levit (1996)):

$$
f_{\theta}(y)=f_{\theta}\left(y, x, \phi_{n}, f_{0}\right)=f_{0}(y)\left[1+\theta\left\{\phi_{n}(x-y)-\bar{\phi}_{n}(x)\right\}\right],
$$

where $|\theta| \leqslant \theta_{n}, \phi_{n}(y)$ is defined by (5) and $\bar{\phi}_{n}(x)=\int \phi_{n}(x-y) f_{0}(y) \mathrm{d} y$. Let $\theta_{n}$ be such that $\epsilon_{n} \leqslant \theta_{n} \leqslant \rho_{n}$, where the positive sequences $\epsilon_{n}$ and $\rho_{n}$ satisfy

$$
\frac{\epsilon_{n}^{-2}}{n \log n}=o(1), \quad \rho_{n}^{2} n=o(1) .
$$

One can choose for example $\theta_{n}=n^{-1 / 2}(\log n)^{-1 / 4}$.

The proof of the theorem will proceed via the following two claims.

Proposition 1. For sufficiently large $n, f_{\theta} \in V$.

Proof. Take $\epsilon>0$ such that $O_{\epsilon}\left(f_{0}\right) \subseteq V$, where

$$
O_{\epsilon}\left(f_{0}\right)=\left\{f \in \tilde{\mathscr{F}}_{\delta}: \rho\left(f, f_{0}\right)<\epsilon\right\} .
$$

We prove now that $f_{\theta} \in O_{\epsilon}\left(f_{0}\right)$ for sufficiently large $n$.

It is easy to check the condition on the nonparametric class (see (4)); for sufficiently large $n$,

$$
\inf _{y}\left[\mathrm{e}^{\tau_{0} y^{2 m}}\left\{1-F_{\theta}(y)\right\}\{1-G(y)\}\right]=\inf _{y}\left[\mathrm{e}^{\tau_{0} y^{2 m}}\left\{1-F_{0}(y)\right\}\{1-G(y)\}\right]\{1+o(1)\}>\alpha
$$

since $F_{0} \in \tilde{\mathscr{F}}_{\delta}$.

Denote

$$
\psi(t)=\psi(t, x)=f_{0}(y) \phi_{n}(x-y) .
$$

First, by the Minkowski inequality and the definition (5) and (6) of the kernel $\phi_{n}$, 


$$
\begin{aligned}
\rho\left(f_{\theta}, f_{0}\right)= & |\theta|\left(\int \cosh ^{2}(\delta t)\left|\hat{\psi}(t, x)-\bar{\phi}_{n}(x) \hat{f}_{0}(t)\right|^{2} \mathrm{~d} t\right)^{1 / 2} \\
& +|\theta| \int\left|f_{0}(y)\left(\phi_{n}(x-y)-\bar{\phi}_{n}(x)\right)\right| \mathrm{d} y \\
\leqslant & 2 \theta_{n}\left(\int \cosh ^{2}(\delta t)|\hat{\psi}(t, x)|^{2} \mathrm{~d} t\right)^{1 / 2}+2 \theta_{n}\left|\bar{\phi}_{n}(x)\right|\left(\int \cosh ^{2}(\delta t)\left|\hat{f}_{0}(t)\right|^{2} \mathrm{~d} t\right)^{1 / 2} \\
& +\theta_{n} \int\left|f_{0}(y)\left\{\phi_{n}(x-y)-\bar{\phi}_{n}(x)\right\}\right| \mathrm{d} y \\
\leqslant & 2 \theta_{n}\left(\int \cosh ^{2}(\delta t)|\hat{\psi}(t, x)|^{2} \mathrm{~d} t\right)^{1 / 2}+C_{1} \theta_{n} \log n .
\end{aligned}
$$

So it suffices to show that the first term on the right-hand side of the last inequality converges to zero as $n \rightarrow \infty$. Since

$$
\hat{\psi}(t, x)=(2 \pi)^{-1} \int \mathrm{e}^{i x u} \hat{f}_{0}(t+u) \hat{\phi}_{n}(u) \mathrm{d} u,
$$

by the generalized Minkowski inequality (Nikol'skii 1975, p. 20), (4), (7) and (9) it follows that

$$
\begin{aligned}
\left(\int \cosh ^{2}(\delta t)|\hat{\psi}(t, x)|^{2} \mathrm{~d} t\right)^{1 / 2} & \leqslant C_{2}\left(\int\left|\int \mathrm{e}^{\delta|t|} \mathrm{e}^{\mathrm{i} x u} \hat{f}_{0}(t+u) \hat{\phi}_{n}(u) \mathrm{d} u\right|^{2} \mathrm{~d} t\right)^{1 / 2} \\
& \leqslant C_{2} \int\left(\int\left|\mathrm{e}^{\delta|t|} \mathrm{e}^{\mathrm{i} x u} \hat{f}_{0}(t+u) \hat{\phi}_{n}(u)\right|^{2} \mathrm{~d} t\right)^{1 / 2} \mathrm{~d} u \\
& \leqslant C_{2} \int\left(\int\left|\mathrm{e}^{\delta|t+u|} \hat{f}_{0}(t+u) \mathrm{e}^{\delta|u|} \hat{\phi}_{n}(u)\right|^{2} \mathrm{~d} t\right)^{1 / 2} \mathrm{~d} u \\
& \leqslant C_{3}\left(\int \cosh ^{2}(\delta t)\left|\hat{f}_{0}(t)\right|^{2} \mathrm{~d} t\right)^{1 / 2} \int \mathrm{e}^{\delta|u|}\left|\hat{\phi}_{n}(u)\right| \mathrm{d} u \\
& \leqslant C_{4} \int \mathrm{e}^{\delta|u|}\left|\hat{\phi}_{n}(u)\right| \mathrm{d} u \\
& \leqslant C_{4}\left(\int \mathrm{e}^{2 \delta|u|}\left|\left(\hat{r} * I_{\left(-a_{n}, a_{n}\right)}\right)(u)\right|^{2} \mathrm{~d} u\right)^{1 / 2} \\
& =C_{4}\left(\int\left|\int \mathrm{e}^{\delta|u|} \hat{r}(u-t) I_{\left(-a_{n}, a_{n}\right)}(t) \mathrm{d} t\right|^{2} \mathrm{~d} u\right)^{1 / 2}
\end{aligned}
$$




$$
\begin{aligned}
& \leqslant C_{4} \int\left(\int\left|\mathrm{e}^{\delta|u|} \hat{r}(u-t) I_{\left(-a_{n}, a_{n}\right)}(t)\right|^{2} \mathrm{~d} u\right)^{1 / 2} \mathrm{~d} t \\
& \leqslant C_{5}\left(\int \mathrm{e}^{2 \delta|t|}|\hat{r}(t)|^{2} \mathrm{~d} t\right)^{1 / 2} \int \mathrm{e}^{\delta|u|} I_{\left(-a_{n}, a_{n}\right)}(u) \mathrm{d} u \\
& \leqslant C_{6}\left(\int \exp \left(2 \delta|t|-2 A_{2}|t|^{2 m /(2 m-1)}\right) \mathrm{d} t\right)^{1 / 2} \int \mathrm{e}^{\delta|u|} I_{\left(-a_{n}, a_{n}\right)}(u) \mathrm{d} u \\
& \leqslant C_{7} \int \mathrm{e}^{\delta|u|} I_{\left(-a_{n}, a_{n}\right)}(u) \mathrm{d} u \\
& \leqslant C_{8} n^{1 / 2} .
\end{aligned}
$$

Recalling the condition on the $\theta_{n}$, we obtain finally that

$$
\rho\left(f_{\theta}, f_{0}\right) \leqslant C_{9} \theta_{n} n^{1 / 2}+C_{1} \theta_{n} \log n \leqslant C_{9} \rho_{n} n^{1 / 2}+C_{1} \rho_{n} \log n=o(1)
$$

as $n \rightarrow \infty$.

If $X_{i}$ is distributed with density $f_{\theta}(y)$, then the corresponding observation $\left(Z_{i}, \Delta_{i}\right)$ has the density

$$
f_{\theta}(y, \tau)=\left[f_{\theta}(y)\{1-G(y)\}\right]^{\tau}\left[g(y)\left\{1-F_{\theta}(y)\right\}\right]^{1-\tau}, \quad \tau \in\{0,1\} .
$$

The following proposition describes the Fisher information $I(\theta)$ about $\theta$ contained in the observation $(Z, \Delta)$.

Proposition 2. As $n \rightarrow \infty$, the relation

$$
I(\theta) \stackrel{\text { def }}{=} \mathrm{E}\left(\frac{\partial\left\{\log f_{\theta}(Z, \Delta)\right\}}{\partial \theta}\right)^{2}=\frac{f_{0}(x)\{1-G(x)\} \log n}{2 \pi \delta}\{1+o(1)\}
$$

holds uniformly in $\theta,|\theta|<\theta_{n}$.

Proof. By straightforward calculations,

$$
\begin{aligned}
I(\theta)= & \mathrm{E}\left(\frac{\partial\left\{\log f_{\theta}(Z, \Delta)\right\}}{\partial \theta}\right)^{2} \\
= & \int \frac{\left\{\phi_{n}(x-y)-\bar{\phi}_{n}(x)\right\}^{2} f_{0}(y)\{1-G(y)\} \mathrm{d} y}{1+\theta\left(\phi_{n}(x-y)-\bar{\phi}_{n}(x)\right)} \\
& +\int \frac{\left[\int_{-\infty}^{y} f_{0}(u)\left\{\phi_{n}(x-u)-\bar{\phi}_{n}(x)\right\} \mathrm{d} u\right]^{2} \mathrm{~d} G(y)}{1-F_{\theta}(y)} .
\end{aligned}
$$

We split the second term on the right-hand side of the last inequality into two parts: the integral over $(-\infty, x+\epsilon]$ and the integral over $(x+\epsilon, \infty)$. If we bound the integrands of 
both integrals, then we obtain in fact that the second term in the last inequality is of order $O(1)$. Since, according to Lemmas 4 and $5, \int_{-\infty}^{y} f_{0}(z)\left\{\phi_{n}(x-z)-\bar{\phi}_{n}(x)\right\} \mathrm{d} z$ is bounded and

$$
1-F_{\theta}(y)=\left\{1-F_{0}(y)\right\}\{1+o(1)\} \geqslant\left\{1-F_{0}(x+\epsilon)\right\}\{1+o(1)\}
$$

for $y \in(-\infty, x+\epsilon]$, the integral over $(-\infty, x+\epsilon]$ is bounded. Note that

$$
\int_{-\infty}^{y} f_{0}(z)\left\{\phi_{n}(x-z)-\bar{\phi}_{n}(x)\right\} \mathrm{d} z=-\int_{y}^{\infty} f_{0}(z)\left\{\phi_{n}(x-z)-\bar{\phi}_{n}(x)\right\} \mathrm{d} z
$$

and the function $\phi_{n}(x-y)$ is bounded for $y \in(x+\epsilon, \infty)$. Therefore, for sufficiently large $n$,

$$
\begin{aligned}
\frac{\left[\int_{-\infty}^{y} f_{0}(z)\left\{\phi_{n}(x-z)-\bar{\phi}_{n}(x)\right\} \mathrm{d} z\right]^{2}}{1-F_{\theta}(y)} & =\frac{\left[\int_{y}^{\infty} f_{0}(z)\left\{\phi_{n}(x-z)-\bar{\phi}_{n}(x)\right\} \mathrm{d} z\right]^{2}}{1-F_{\theta}(y)} \\
& \leqslant \frac{C_{1}\left\{\int_{y}^{\infty} f_{0}(z) \mathrm{d} z\right\}^{2}}{1-F_{\theta}(y)} \\
& =\frac{C_{1}\left\{\int_{y}^{\infty} f_{0}(z) \mathrm{d} z\right\}^{2}}{\left\{1-F_{0}(y)\right\}\{1+o(1)\}} \\
& \leqslant C_{2}\left\{1-F_{0}(y)\right\} \\
& \leqslant C_{2}
\end{aligned}
$$

for $y \in(x+\epsilon, \infty)$. Thus, we obtained that

$$
\int \frac{\left[\int_{-\infty}^{y} f_{0}(z)\left\{\phi_{n}(x-z)-\bar{\phi}_{n}(x)\right\} \mathrm{d} z\right]^{2} \mathrm{~d} G(y)}{1-F_{\theta}(y)}=O(1)
$$

uniformly in $\theta,|\theta|<\theta_{n}$. According to Lemmas 3 and 4 , it is not difficult to see that

$$
\int \frac{\left\{\phi_{n}(x-y)-\bar{\phi}_{n}(x)\right\}^{2} f_{0}(y)\{1-G(y)\} \mathrm{d} y}{1+\theta\left\{\phi_{n}(x-y)-\bar{\phi}_{n}(x)\right\}}=\frac{f_{0}(x)\{1-G(x)\} \log n}{2 \pi \delta}\{1+o(1)\}
$$

uniformly in $\theta,|\theta|<\theta_{n}$. Relation (27) is proved.

Now we proceed to prove the theorem. Introduce

$$
v(x)=v_{n}(x)=\theta_{n}^{-1} v_{0}\left(\theta_{n}^{-1} x\right),
$$

where $v_{0}(x)$ is a probability density on the interval $[-1,1]$ with finite Fisher information 


$$
I_{0}=\int_{-1}^{1}\left\{v_{0}^{\prime}(x)\right\}^{2} v_{0}^{-1}(x) \mathrm{d} x,
$$

such that $v_{0}(-1)=v_{0}(1)=0$ and $v_{0}(x)$ is continuously differentiable for $|x|<1$. The function $v(x)$ is the probability density with support $\left[-\theta_{n}, \theta_{n}\right]$. It is easy to calculate the Fisher information of the distribution defined by the density $v(x)$ :

$$
I(v)=I_{n}(v)=I_{0} \theta_{n}^{-2} .
$$

Applying now the van Trees inequality for the Bayes risk below (van Trees 1968, Gill and Levit 1995, Golubev and Levit 1996) and Propositions 1 and 2, we obtain that, for sufficiently large $n$,

$$
\begin{aligned}
r_{n}(V) & =\inf _{\tilde{f}_{n}} \sup _{f \in V} \mathrm{E}\left\{\tilde{f}_{n}-f(x)\right\}^{2} \\
& \geqslant \inf _{\tilde{f}_{n}} \sup _{|\theta| \leqslant \theta_{n}} \mathrm{E}_{f_{\theta}}\left\{\tilde{f}_{n}-f_{\theta}(x)\right\}^{2} \\
& \geqslant \inf _{\tilde{f}_{n}} \int \mathrm{E}_{f_{\theta}}\left\{\tilde{f}_{n}-f_{\theta}(x)\right\}^{2} v(\theta) \mathrm{d} \theta \\
& \geqslant \frac{\left[\int\left\{\partial f_{\theta}(x) / \partial \theta\right\} v(\theta) \mathrm{d} \theta\right]^{2}}{n \int I(\theta) v(\theta) \mathrm{d} \theta+I(v)} \\
& =\frac{\left\{f_{0}(x) \phi_{n}(0)\right\}^{2}}{n \int I(\theta) v(\theta) \mathrm{d} \theta+I_{0} \theta_{n}^{-2}} \\
& \geqslant \frac{\left[\left\{f_{0}(x) \log n\right\} / 2 \delta \pi\right]^{2}}{n\left(\left[f_{0}(x)\{1-G(x)\} \log n\right] / 2 \pi \delta\right)\{1+o(1)\}+I_{0} \epsilon_{n}^{-2}} \\
& \geqslant \frac{f_{0}(x) \log n\{1+o(1)\}}{2 \pi \delta\{1-G(x)\} n}
\end{aligned}
$$

or

$$
\liminf _{n \rightarrow \infty} \frac{n}{\log n} r_{n}(V) \geqslant \frac{f_{0}(x)}{2 \pi \delta\{1-G(x)\}}=\sigma^{2}\left(f_{0}\right) .
$$

The function $f_{0}$ was chosen arbitrarily from the neighbourhood $V$ and hence, by the same reasoning, this relation is valid for any function $f \in V$ :

$$
\liminf _{n \rightarrow \infty} \frac{n}{\log n} r_{n}(V) \geqslant \sigma^{2}(f) \text {. }
$$

Therefore

$$
\liminf _{n \rightarrow \infty} \frac{n}{\log n} r_{n}(V) \geqslant \sup _{f \in V} \sigma^{2}(f),
$$

which proves Theorem 2 . 


\section{Acknowledgement}

The author is grateful to B. Levit for helpful conversations and suggestions.

\section{References}

Andersen, P.K., Borgan, O., Gill, R.D. and Keiding, N. (1993) Statistical Models Based on Counting Processes. New York: Springer-Verlag.

Diehl, S. and Stute, W. (1988) Kernel density and hazard function estimation in the presence of censoring. J. Mult. Anal., 25, 299-310.

Fedoruk, M.V. (1977) Metod Perevala. Moscow: Nauka. (In Russian.)

Gill, R.D. (1980) Censoring and Stochastic Integrals, Vol. 124. Amsterdam: Mathematisch Centrum.

Gill, R.D. and Levit, B.Y. (1995) Applications of the van Trees inequality: a Bayesian Cramer-Rao bound. Bernoulli, 1, 59-79.

Golubev, G.K. and Levit, B.Y. (1996) Asymptotically efficient estimation for analytic distributions. Math. Methods Statist., 5, 357-368.

Gradshtein, I.S. and Ryzhik, I.M. (1980) Table of Integrals, Series, and Products. New York: Academic Press.

Hentzschel, J. (1992) Density estimation with Laguerre series and censored samples. Statistics, 23, $49-61$.

Huang, J. and Wellner, J.A. (1995) Estimation of a monotone density and monotone hazard under random censoring. Scand. J. Statist., 22, 3-33.

Ibragimov, I.A. and Hasminskii, R.Z. (1983) Estimation of distribution density. J. Sov. Math., 25, 40-57. (Originally published in Russian in 1980.)

Katznelson, Y. (1976) An Introduction to Harmonic Analysis, 2nd corrected edn. New York: Dover Publications.

Kulasekera, K.B. (1995) A bound on the $L_{1}$-error of a nonparametric density estimator with censored data. Statist. Probab. Lett., 23, 233-238.

Liu, R.C. (1996) Optimal rates, minimax estimations, and $\mathrm{K}-\mathrm{M}$ based procedures for estimating functionals of a distribution under censoring. Technical Report, Department of Mathematics, Cornell University.

Lo, S.H., Mack, Y.P. and Wang, J.L. (1989) Density and hazard rate estimation for censored data via strong representation of the Kaplan-Meier estimator. Probab. Theory Related Fields, 80, 461-473.

Mielniczuk, J. (1986) Some asymptotic properties of kernel estimators of a density function in case of censored data. Ann. Statist., 14, 766-773.

Nikol'skii, S.M. (1975) Approximation of Functions of Several Variables and Imbedding Theorems. Berlin: Springer-Verlag.

Timan, A.F. (1963) Theory of Approximation of Functions of a Real Variable. Oxford: Pergamon. van Trees, H.L. (1968) Detection, Estimation and Modulation Theory, Part 1. New York: Wiley.

Weits, E. (1993) The second order optimality of a smoothed Kaplan-Meier estimator. Scand. J. Statist., 20, 111-132.

Yang, S. (1994) A central limit theorem for functionals of the Kaplan-Meier estimator. Statist. Probab. Lett., 21, 337-345.

Received March 1996 and revised April 1997 